\title{
GRAPHICAL REPRESENTATION IN TUTORING SYSTEMS
}

\author{
Nabila Khodeir \\ Informatics Department, Electronics Research Institute, Cairo, Egypt
}

\begin{abstract}
Visual representation and organization of the knowledge have been utilized in different ways in tutoring systems to upgrade their usefulness. This paper concentrates on the usage of various graphical formalisms, for example, the conceptual graph, ontology, and concept map in tutoring systems. The paper addresses what is way of the utilization of every formalism and the offering of the potential outcomes to assist the student in education systems.
\end{abstract}

\section{KEYWORDS}

Conceptual graph, ontology, concept map.

\section{INTRODUCTION}

The graphical representation is based on linking some concepts to each other by semantic relations. Conceptual graph, ontology, and concept map are the most common formalisms for the description of such graphical structure. Each formalism has its own characteristics that are selected for a particular use in the learning setting.

A conceptual graph is a finite, connected, undirected graph with two types of nodes. First, one is called concepts and the other type called conceptual relations. The conceptual graph is composed of propositions defined by two concept nodes and one connecting relation link [1]. The main application of conceptual graph in the learning context is to represent the prerequisite relations between domain concepts to be utilized in check out the origin of the student errors.

Ontology is an explicit formal specification of types and properties of the domain terms and relations among them [2,3]. Developing ontologies in the learning context aims to share a common understanding of the structure of information among people or software agents [4,2]. That enables the reuse of a domain knowledge which allows building a large ontology by integrating several existing ontologies.

A concept map is a graphical tool for organizing and representing knowledge which was first introduced by Novak and Gowin [5]. It is based on representing the main ideas or concepts as nodes and linking between them by relations. The unit that has a node-link-node connection is called a proposition or semantic unit, or unit of meaning [5]. Hierarchical representation is usually used in concept map where the most general concepts at the top and more specific nodes arranged hierarchically below. Representation of the knowledge using the concept map starts by defining the context for it within a specific domain. Then, key concepts that apply to this domain are defined and related to each other by links that encode relationships in the domain to constitute the preliminary concept map [6]. A more advanced step that requires high levels of cognitive performance, namely evaluation and synthesis of knowledge [7], is to define cross-links which are links between concepts in different segments of knowledge on the map [6]. Concept map supports meaningful learning that aims to relate the new knowledge to the relevant concepts 
already known to the student [5]. Concept maps have been used in learning contexts as a learning and evaluation tools [8,9].

We separate the use of graphical representation to three classes i) representation of the domain or the learning material, ii) diagnosing tool for the student errors, and the iii) assessing the student visual expression of a specific part of domain concepts. Distinctive uses are because of the diverse description of the domain concepts and the way of the semantic relations between these concepts. The paper will investigate the distinctive usage of the graphical representations through these categories.

The rest of the paper is organized as follows. Section 2 investigates using of the graphical representation as a domain model. Diagnosing the student errors based on historical test scores and how to guide the student through the learning process is addressed in section 3 . Section 4 focuses on the concept mapping technique that assessing the student graphical expressions of his/her knowledge. Finally, Section 5 provides the conclusion of this work.

\section{GRAPHICAL REPRESENTATION AS A DOMAIN MODEL}

The domain model is an integral part in intelligent educational applications. Ontology and concept map are used in representing the learning material. The concept map is used in limited intelligent tutoring systems as a domain model to be used as a reference in the modeling of the student knowledge. Other systems automatically extracting concept maps from different resources to be used in automatic question generation or to extract and organize the domain concepts in a hierarchal order. On the other hand, ontology formalization is used to represent a sharable domain on the web. Different features are represented for the learning materials to be used in adaptive hypermedia techniques. Next sections will investigate using the concept map and ontology in domain representation.

\subsection{Concept Map as a Domain Model}

As we mentioned limited intelligent tutoring systems use the concept map as a domain model to assess the student knowledge through the different concepts. For instance; Kordaki and Psomos [10] present an intelligent concept mapping tool to assess the student knowledge and diagnosing and treat his misconceptions. An interactive questionnaire is attached to each node of the concept map to evaluate students' knowledge. Subsequently, the system automatically providing appropriate personalized feedback for each learner to diagnose their mistakes according to the answers given by each student. The system creates an adapted version of the concept map for each individual student by adding appropriate statistical data to each of its nodes and colors to indicate the student knowledge status.

Another example is presented by Kumar [11] who presents intelligent tutors for programming that uses the concept map as a domain model in addition to using an overlay of it as a student model. To obtain a finer-grained student model pedagogical concepts called learning objectives are added for each correct concept in the domain. Moreover, potential errors associated with each concept is represented as a separate learning objective. Using the concept map representation as an overlay student model has the advantage of allowing individual assessment of each concept to influence related concepts. In addition, it primates focusing on specific concepts in the student model for more efficient assessment. The student model is used to adapt the selection of the presented problems where learning objectives in the student model that have not yet been met are enumerated. Then the next problem is selected such that the problem addresses one or more of these learning objectives. After the student solves each problem, the tutor updates credit for all the affected learning objectives in the student model and recalculates the set of learning objectives that remain to be met. 
Some systems generate the domain model concept map automatically from different resources. Olney et al., [12,13] present a methodology for automatically extracting concept maps from textbooks using term extraction, semantic parsing, and relation classification. The methodology is based on SemNet formulation [14] that take the form of one layer of links radiating out of a core concept. The generated concept map consists of fragments that are called triples. Each triple has to start by a node contains key term (pedagogically significant terms in the domain) and end by another node that can contain key terms, other words, or complete propositions. Labeled edges are used to connect between the two nodes. Since Only et al. [12,13], aim to utilize the generated concept map in generating questions and answers, they use restricted set of labeled edges to facilities that target. The index and glossary are used as sources of getting the key terms. Automatic extraction for the triples starts by using LTH SRL parser to get information about the word token's part of speech, lemma, head, and relation to the head. After parsing, four triple extractor algorithms are applied to each sentence. Each extractor first attempts to identify a key term as a possible start node. After triples are extracted from the parse, they are filtered to remove triples that are not particularly useful for generating concept map exercises. The final filter uses likelihood ratios to establish whether the relation between start and end nodes is meaningful.

A concept hierarchy is neglected in [12,13] although it is a powerful tool for representing and organizing the domain knowledge. In addition to utilizing it in diagnosing the cause of the student errors and assessing of the student knowledge. Wang et al., [15] deal with extracting the concept hierarchy from Textbooks using the lexical content and table of contents (TOC). In addition, they augment Web knowledge and extracts a set of related important Wikipedia concepts for each book chapter and organize them as a concept hierarchy using the book's TOC. Learning-to-Rank approach is used to extract concept hierarchy which considers both local relatedness and global coherence. They propose three sets of global features, which guarantee less redundancy, consistency and appropriate learning order for a concept hierarchy that captures the global coherence embedded in a book. They first extract a domain-specific dictionary for a given book topic and then performs candidate selection for each chapter. Finally, by re-ranking the candidates based on the local and global features, it generates the concept hierarchy which arrives at coherent sets of important concepts for a given book.

Wang et al., [16] focus on the construction of prerequisite concept maps to discover students' learning gaps and work on closing these gaps. They implemented a two-phase model that includes domain concepts extraction and prerequisite relationships identification. They start by constructing a domain-specific concept dictionary in which each concept is the title of a domain related Wikipedia page. Then given an article, they identify all Wikipedia concepts in the article using this dictionary and obtain a list of Wikipedia candidates.

\subsection{Ontology as a Domain Model}

Ontology formalism is usually used in web-based systems to describe the learning material or the learning objects. The learning object is anything digital that can be delivered across the network on demand such as text, images, applets, and entire web pages. Gascueña et al. [17] consider two characteristics to define each learning object, the most appropriate learning style and the most satisfactory hardware and software features of the used device. A questionnaire is used to found the dominant learning style of each student. Based on these features and the student learning style adaptive e-learning environments and reusable educational resources are provided. Chi et al. [18] present another example that utilizes semantic rules in combination with ontologies to model curriculum contents sequencing expertise into a knowledge. The ontology was used to represent abstract views of content sequencing and course materials and semantic rules were used to express relationships between individuals. 
Due to rapid increase of learning content on the Web, it will be time-consuming for learners to find adequate learning material. Yu et al. [19] present an ontology-based approach for semantic content recommendation to realize context-awareness in e-learning. The approach is recommending of a learning material based on considering knowledge about the learner, knowledge about content, and knowledge about the domain being learned. Ontology is utilized to model and represent such kinds of knowledge. The recommendation approach proceeds in four steps. First, the Semantic Relevance Calculation computes the semantic similarity between the learner and the learning contents to generate a recommendation list. Second, the Recommendation Refining provides an interactive way to allow the learner to select one item from the candidates, the Learning Path Generation guides the learning process by builds a studying route composed of prerequisite contents and the target learning contents. Finally, the Recommendation Augmentation aggregates appending contents related with the main course.

Vesin et al. [20] present programming tutoring system "Protus" which rely on Semantic web standards and technologies. Implemented architecture utilizes ontology, where the representation of each component is made by a specific ontology. This allows interoperability and reusability of the system in addition to the communication among the different components. The system contains domain ontology that represents types of all essential learning materials. The different types support the different learning style of the users. The role of each specific resource from domain ontology is represented in the task ontology. The learner personal information, learning style and performance constitute the learner model ontology. Teaching strategy ontology consists of selecting or computing a specific navigation sequences among the resources. The decisions are drawn on the basis of the information in learner model ontology, task ontology, and domain ontology. Most appropriate learning pattern or resource that will be recommended to the learner is selected. Finally, the interface ontology is used to reads a decision from the Teaching strategy ontology, and based on that decision it creates navigation sequence of resources recommended for a specific learner and generates an interface view to the learner.

\section{DiGNOSING}

Defining of the prerequisite relations between different concepts in the conceptual graph gives the potential to guide the student on concepts that needing improvement and the path or sequence to learning. Hsu et al. [21] proposed a concept effect relationship (CER) model which proves how certain concepts are a perquisite to efficiently performing other concepts. Some systems utilize the conceptual graphs method for modeling the prerequisite relationships among the domain concepts to be learned. Then, the student test results are analyzed based on such conceptual graphs. Jong et al. [22] present an algorithm for diagnosing individual student learning situation based on predefined weighted conceptual graphs. The algorithm has the potential to providing the Remedial-Instruction Decisive path (RID path) that identify the student's missing concepts. Student learning situation that defines if the students have mastered certain concept is estimated based on the Sequential Probability Radio Test (SPRT) [23]. The algorithm of the RID path finds a remedial instruction decisive path based on missing concepts using SPRT. Once a certain student is assessed to have failed to achieve mastery of a certain concept, their prior concepts of that student can be obtained through the conceptual graphs. These concepts are verified to detect the missing prior concepts. The algorithm repeats the diagnosis steps for each missing prior concept. Finally, all missing concept nodes are obtained which represented the RID. Each student has a distinctive evaluated conceptual graph which maps his knowledge structure and can be considered as an overlay model.

Since it is time-consuming for teachers to build a conceptual graph that includes the prerequisite relationships between concepts, some system automates this process based on long-term analysis of the students' test items status or score. Hwang et al. [24] present an algorithm that starts by 
finding the test item that most students failed to answer correctly, then finds the other test items that were incorrectly answered by those students, and finally uses this information to determine the relationships among the test items. The relationships among concepts can consequently be determined based on the relationships among test items, and between test items and concepts. Getting the Concept effect relationships are refined by using the support and belief values which are two thresholds. Many noisy relationships may be generated if the thresholds approach 0 , while some important relationships may be missed if the thresholds approach 1 . The most appropriate support and belief values for generating concept effect relationships based on the outcome of previous applications. Data mining techniques proposed in [25] are used to explore the relationships among some attributes, and the relationships identified are then employed to assist in determining belief and support values for future applications.

Hsu et al. and Hwang et al [21,24] focus on single rule type, L-L type, which means students get a low grade on specific question implies that they may also get a low grade on specific another question, which may decrease the quality of concept map. On the other hand, Tseng et al. [26] propose a Two-Phase Concept Map Construction (TP-CMC) algorithm to automatically construct a concept map of a course by historical testing records. They apply Fuzzy Set Theory to transform the numeric testing records of learners into symbolic in the first phase. Then data mining approach is applied to find its grade fuzzy association rules. The mined grade fuzzy association rules include four rule types, $\mathrm{L}-\mathrm{L}, \mathrm{L}-\mathrm{H}, \mathrm{H}-\mathrm{L}$, and $\mathrm{H}-\mathrm{H}$, which denote the casual relations between learning concepts of quizzes for all types of grades (Low or High).

\section{CONCEPT MAPPING}

Concept mapping is a supportive way in learning. That stem from the fact that people understand and remember the knowledge after they organize and integrate it [27]. Moreover, it is easy for people to memorize and recall the ideas which are correlative [28]. Concept mapping serves as a kind of template or scaffold to help to organize knowledge and to structure it [6]. In addition, Concept mapping has been introduced as an effectively visualized learning tool that helps learners memorize and organize their knowledge [5].

In e-learning context, concept mapping technique is used for the student to express visually his/ her understanding of the domain concepts in terms of concepts that are related by hierarchy relationships. It is used as an assessment tool which is characterized in terms of the directedness that is provided for the student to express his/her knowledge structure, the student response and the scoring mechanism to evaluate the student concept's map [29]. It also could be considered as a visual form of the student model that can be utilized to assess the student's knowledge about a specific domain and to be used in adapting of the learning system resources.

Concept mapping is a challenging task where it requires the student to reflect his understanding of the concepts and their interrelations [5]. In order to guide the students, concept mapping assessment tools use a less free-form approach to mapping. [29] Identifies a scale from low to high directedness in concept mapping tools based on the provided information to the students. High-directed concept map tasks support students with a template of the concept map and the students asked to fill some missing concepts or relations. On the other hand, in a low-directed concept map tasks, students are free to construct their maps and select the concepts, relations, and structure [30].

The assessment mechanism is usually based on comparing learner's map with the predefined expert's map [29]. It has two main methods which are the structural method and the relational method. The structural method [5] is restricted on hierarchical maps and considers the valid map components such as propositions, and links. On the other hand, the relational method focuses on the accuracy of each proposition [29], [31]. 
In the following sections, we will explore a number of systems to show the directedness level, scoring mechanism, presented guidance and if they have adaptation mechanism.

\subsection{Structural Method Based Assessment Systems}

Chang et al. [32] presented a system that provides two learning environments. First one is the 'construct-by-self' environment, the system provides students with the evaluation results and corresponding hints for feedback. The students construct concept maps by themselves with only the assistance of the feedback. In the 'construct-on scaffold' environment, in addition to the feedback, the students receive an incomplete concept map, within which some nodes and links were set as blanks for the scaffold. A study comparing the effectiveness of the 'construct-by-self', 'construct-on-scaffold', and 'construct by paper-and-pencil' concept mapping showed that the 'construct-on-scaffold' had a better effect for learning on biology. Scoring the student concept map is based on comparing the numbers of valid propositions, valid hierarchical levels, and valid cross-links. The score for a student concept map is divided by the score of an expert concept map to produce a ratio as the similarity index. For the 'construct-on-scaffold' version, the similarity index of a map is estimated by getting the ratio between the number of correct answers in the blanks and the number of total blanks on the map. Both of the two indices range from zero to one. Zero shows that the two maps are completely different. One point to that the two maps are identical.

Cimolino et al. [33] present a verified concept mapper where the students create a map from a given list of concepts and links which are predefined by the teacher. The student map is interpreted by the teacher to model the student understanding in terms of saved sentences. Such sentences are shown to the students to indicate what information in their current map is being saved to their user model. In addition, questions are provided in case of student errors to guide him in correcting them. For example, the default question associated with a missing concept is to ask the student if they can see how to include it on the map.

Hwang et al. [34] integrate concept mapping in educational computer game aiming to help students to organize what they have learned during the game-based learning process. Such a concept map-embedded gaming approach made the students highly accepting of the appearance and assistance of the concept maps during the gaming process. The system includes concept mapping module to assists the students in organizing the collected data following the storyline based on the concept map templates provided by the teachers.

Jain et al. [35] present an artificial intelligence-based student learning evaluation tool (AISLE) to evaluate student learning using concept maps. The student would be given a topic to learn and build a concept map based on their understanding of the topic. Which means concepts maps are developed by the students from the scratch. The implemented scoring system is based on the structure of the concept map where scores are assigned in the form of numbers to every concept that is presented in the hierarchy of concept. The hierarchy is included in the scoring to give the level of the student understands of the topic in the study. Z-score for each concept is used as standardization of scores which is used as a function to estimate the probability distribution for all concepts in the hierarchy of concepts. The standard probability distribution of the curve is used as a reference curve to evaluate the concept maps drawn by the students. The concept map drawn by the students is verified and validated by the instructor.

Leelawong et al. [36,37] have designed learning environments where students teach a computer agent, called a Teachable Agent (TA). The concept map is used as a visual representation to help structure domain knowledge. In addition reasoning through the concept map links are considered where TAs can show their reasoning based on how they have been taught which helps students to assess their teaching. Scoring mechanism is based on comparing the student map with the expert 
map. Concepts and links were labeled as "expert" if they were in the expert map while concepts and links that were not in the expert map, but were resulted from the correct understanding of the domain were graded as "relevant." The number of valid concepts and links in a student's map was the sum of the expert and relevant concepts and links.

Sormo [38] presents CREEK-Tutor, an exercise oriented tutoring system that uses a student modeling technique based on case-based reasoning to find students of similar competence. Firstly, concepts and linking names are extracted from the teacher concept map for a topic. Then the student is asked to construct his map using the same concepts and link names. This approach is different from previous approaches in that the goal is not to score the student's concept map by its similarity to the teacher's map but to use it to find students that are similar in ability. The similarity is measured by finding the difference between the union and intersection between two graphs. Two main contributions are presented in CREEK-Tutor. Firstly, included procedural knowledge with the fact knowledge in the concept map by introducing a lot of examples of program code snippets as a correct or wrong example. Second, a lot of traditional programming exercises are combined with constructing the concept map. The student answers are recorded and used as an offline data set that contains for each student a concept map and various measures of how the student performed on each programming task. That is used later in adapting the selection of the presented exercise for each student. The exercise selection algorithm is based considering the student concept map of a particular topic. Then find similar concept maps drawn by other and predict the difficulty of exercises based on the performance of students found to have similar concept maps. Suggest an exercise of appropriate difficulty level and justify the exercise selection by showing which part of the concept map it addresses.

\subsection{Relational Method BaSed Assessment Systems}

Po-Han et al. [39] present an Interactive Concept Map-oriented Learning System which enables learners to construct concept maps in personal computers and share them on servers via the Internet. The system provides immediate evaluation of concept maps and gives also real-time feedback to the students. The scoring mechanism is based on comparing each of student's concept map propositions with the corresponding proposition in the expert's concept map. In a case of matching, the weighting of the proposition is added to the accumulated score for the student's concept map. If the two propositions are partially matched, only half of the weighting is added to the accumulated score. Accordingly, to the evaluation results, the system provides feedback which indicates student errors on the structure of the concept map developed by individual students, such as missing concepts or relations. In addition, learning materials related to the missing or incorrect concepts/connections are provided to the student as supplementary information.

Conlon et al. [40] consider the characterization of a proposition in its assessment were fully correct means full matching between the student proposition and the corresponding of the expert. Partly correct indicates the relationship between two concepts or the direction of the arrow is incorrect. In addition, the weights of each characterization, and the number of valid concepts included in learner's map are considered in the evaluation process.

Gouli et al. [41] propose a scheme that has been embedded in COMPASS, an adaptive web-based concept map assessment tool. The system presents adaptive feedback based on the evaluation of the student knowledge level on the concept of the map. The assessment process is based on comparing the propositions presented on student's map and expert map. Weights are assigned by the teacher to reflect the degree of importance of the concepts and propositions. In addition, different error categories are identified which characterize Compass system to support the adaptive feedback. 


\section{CONCLUSiON}

Different graphical representations formalisms have been used in the learning context. Limited intelligent tutoring systems use the concept map as a domain model to assess the student knowledge through the different concepts. Other systems automatically extracting concept maps from textbooks aiming to generate questions and answers while other systems extract the concepts and the prerequisite relations between them to discover students' learning gaps and work on closing these gaps. Concept mapping technique focuses on assessing of the student visual expression of his/her knowledge. It is a technique for the student to express visually his/ her understanding of the domain concepts in terms of concepts that are related by hierarchy relationships. It is used as an assessment tool which is characterized in terms of the directedness that is provided for the student to express his/her knowledge structure. It also could be considered as a visual form of the student model that can be utilized to assess the student's knowledge about a specific domain and to be used in adapting of the learning system resources.

Some systems utilize the conceptual graphs method for modeling the prerequisite relationships among the domain concepts to be learned. Then, the student test results are analyzed based on such conceptual graphs. Since it is time-consuming for teachers to build a conceptual graph that includes the prerequisite relationships between concepts, some systems automate this process based on long-term analysis of the students' test items status or score

Ontology is used as a formalism to describe knowledge and information in a way that can be shared on the web. Adding more description about the learning style or the role of each knowledge item allows different adaptation techniques to be applied such as adaptive presentation and adaptive navigation.

\section{REFERENCES}

[1] Sowa J.F. (1976) Conceptual graphs for a data base interface. IBM Journal of Research and Development 20, 336-357.

[2] Gruber, T.R. (1993). A Translation Approach to Portable Ontology Specification. Knowledge Acquisition 5: 199-220.

[3] Gascueña, J. M., Fernández-Caballero, A. \& González, P. (2006). Domain ontology for personalized e-learning in educational systems. In The Sixth IEEE international conference on advanced learning technologies (pp. 456-458).

[4] Musen, M.A. (1992). Dimensions of knowledge sharing and reuse. Computers and Biomedical Research 25: 435-467.

[5] Novak, J.D. and D.B. Gowin, Learning how to learn. 1984: Cambridge University Press.

[6] Novak, J. D. \& A. J. Canas (2008). The Theory Underlying Concept Maps and How to Construct and Use Them. Technical Report IHMC CmapTools 2006-01 Rev 01-2008, Florida Institute for Human and Machine Cognition, 2008.

[7] Bloom, B. S. (1956). Taxonomy of educational objectives; the classification of educational goals (1st ed.). New York: Longmans Green. Pankratius,W. J. (1990). Building an organized knowledge base: concept mapping and achievement in secondary school physics. Journal of Research in Science Teaching, 27(4), 315-333.

[8] Mintzes, J. J., Wandersee, J. H., \& Novak, J. D. (2000). Assessing science understanding: A human constructivist view. San Diego: Academic Press.

[9] Novak, J. D. (1990). Concept maps and vee diagrams: Two metacognitive tools for science and mathematics education. Instructional Science, 19, 29-52

[10] M. Kordaki, P. Psomos (2014). An intelligent concept mapping tool for the diagnosis and treatment of students' misconceptions. 6th World Conference on Educational Sciences.

[11] Kumar, A. (2006). Using Enhanced Concept Map for Student Modeling in a Model-Based Programming Tutor, in Proc. of 19th International FLAIRS Conference on Artificial Intelligence, Melbourne Beach, FL 
[12] Olney, A., (2010). Extraction of concept maps from textbooks for domain modeling. In: Kay, J., Aleven, V. (Eds.), Proceedings of 10th International Conference on Intelligent Tutoring Systems. Springer,Berlin/Heidelberg, pp. 390-392

[13] Olney, A.M., Cade, W.L., Williams, C.: Generating Concept Map Exercises from Textbooks. In: Proceedings of the Sixth Workshop on Innovative Use of NLP for Building Educational Applications, pp. 111-119. Association for Computational Linguistics, Portland, Oregon (2011)

[14] Fisher, K., Wandersee, J., Moody, D.: Mapping biology knowledge. Kluwer AcademicPub., Dordrecht (2000)

[15] S. Wang, C. Liang, Z. Wu, K. Williams, B. Pursel, B. Brautigam, S. Saul, H. Williams, K. Bowen, and C. Giles. Concept hierarchy extraction from textbooks. The ACM Symposium on Document Engineering, 2015.

[16] S. Wang and L. Liu. Prerequisite concept maps extraction for automatic assessment. In WWW, pages 519-521, 2016

[17] Gascueña, J. M., Fernández-Caballero, A. \& González, P. (2006). Domain ontology for personalized e-learning in educational systems. In The Sixth IEEE international conference on advanced learning technologies (pp. 456-458).

[18] Chi, Y. L. (2009). Ontology-based curriculum content sequencing system with semantic rules. Expert Systems with Applications, 36, 7838-7847.

[19] Yu, Z. et al. (2007). "Ontology-Based Semantic Recommendation for Context-Aware E-Learning." Proc. of the 4th Conference on Ubiquitous Intelligence and Computing, v.4611, Berlin, Heidelberg: Springer, pp. 898-907.

[20] Vesin B., Ivanović M., Klašnja-Milićević A.,Budimac Z., Protus 2.0: OntologyBased Semantic Recommendation in Programming Tutoring System, Experts systems with application, (2012), DOI: 10.1016/j.eswa.2012.04.052.

[21] C. S. Hsu, S. F. Tu, and G. J. Hwang, "A concept inheritance method for learning diagnosis of a network-based testing and evaluation system," The 7th International Conference on ComputerAssisted Instructions, 1998, pp. 602-609.

[22] Jong, BinShyan, Lin, TsongWuu, Wu, YuLung, \& Chan, Teyi (2004). Diagnostic and remedial learning strategy based on conceptual graphs. Journal of Computer Assisted Learning, 20(5), 377386.

[23] Wald A. (1947) Sequential Analysis. Wiley, New York.

[24] Hwang, G. J. (2003). A conceptual map model for developing intelligent tutoring system. Computers and Education, 40(3), 217-235.

[25] J. S. Park, M. S. Chen, and P. S. Yu, "Using a hash-based method with transaction trimming and database scan reduction for mining association rules," IEEE Transactions on Knowledge and Data Engineering, Vol. 9, (1997), pp. 813-825.

[26] S. Tseng, P. Sue, J Su, J Weng, W Tsai: A new approach for constructing the concept map, Computers and Education, Vol 38, pp123-141,2007.

[27] Brainerd, C.J. \& Gordon, L.L. (1994). Development of verbatim and gist memory for numbers. Developmental Psychology, 30(2), 163-177.

[28] Joseph D. N. \& Alberto J. C. (2008). The Theory Underlying Concept Maps and How to Construct and Use Them. Institute for Human and Machine Cognition, 1.

[29] Ruiz-Primo, M. A. \& Shavelson, R. J. (1996). Problems and issues in the use of concept maps in science assessment. Journal of Research in Science Teaching, 33(6), 569-600.

[30] Ruiz-Primo, M.A.: Examining Concept Maps as an Assessment Tool. Proc. Of the FirstInt. Conference on Concept Mapping. Pamplona, Spain, http://cmc.ihmc.us/papers/cmc2004-036.pdf (last access 13.04.05) (2004).

[31] McClure, J., Sonak, B., \& Suen, H. (1999). Concept map assessment of classroom learning: Reliability, validity, and logistical practicality. Journal of Research in Science Teaching, 36, 475-492.

[32] Chang, K., Sung, T., \& Chen, S. (2001). Learning through computer-based concept mapping with scaffolding aid. Journal of Computer Assisted Learning, 17 (1), 21-33.

[33] Cimolino, L., Kay, J., Miller, A. (2003). Incremental student modelling and reflection by verified concept mapping. In Supplementary Proceedings of the AIED2003: Learner Modelling for Reflection Workshop, 219-227.

[34] Hwang, G. J., Yang, L. H., \& Wang, S. Y. (2013). A concept map-embedded educational computer game for improving students' learning performance in natural science courses. Computers \& Education, 69,121-130. 
[35] G. P. Jain, V. P. Gurupur, J. L. Schroeder, and E. D. Faulkenberry, “Artificial intelligence-based student learning evaluation: a concept map-based approach for analyzing a student's understanding of a topic," IEEE Transactions on Learning Technologies, vol. 7, no. 3, pp. 267-279, (2014).

[36] Leelawong, K., \& Biswas, G. (2008). Designing learning by teaching agents: The Betty's Brain system. International Journal of Artificial Intelligence in Education, 18(3), 181-208.

[37] Biswas, G., Segedy, J.R. \& Leelawong, K. (2015). From Design to Implementation to Practice - A Learning by Teaching System: Betty's Brain. International Journal of Artificial Intelligence in Education.

[38] F. Sormo. Case-based student modeling using concept maps. Case-Based Reasoning Research And Development, Proceedings, 3620:492-506, 2005.

[39] Wu, P. H., Hwang, G. J., Milrad, M., Ke, H. R., \& Huang, Y. M. (2011). An innovative concept map approach for improving students' learning performance with an instant feedback mechanism. British Journal of Educational Technology. doi:10.1111/j.1467-8535.2010.01167.x.

[40] Cimolino, L., Kay, J., Miller, A. (2003). Incremental student modelling and reflection by verified concept mapping. In Supplementary Proceedings of the AIED2003: Learner Modelling for Reflection Workshop, 219-227.

[41] Gouli, E, Gogoulou, A., Papanikolaou, K. A., \& Grigoriadou, M. (2005). Evaluating Learner's Knowledge Level on Concept Mapping Tasks. Fifth IEEE International Conference on Advanced Learning Technologies, 424-428.

\section{AUTHORS}

Nabila Khodeir, is a researcher in the Informatics department at the Electronics Research Institute, Cairo, Egypt. Her research interests include intelligent tutoring systems, user modelling and natural language processing. She earned her Ph.D. and ME from the Electronics and Communications department at Cairo University. 